\title{
Intersection of crisis loci in a driven nonlinearly damped oscillator
}

\author{
F. Palmero ${ }^{a}$, F. Romero Romero ${ }^{b}$, J.L. Romero ${ }^{c}$, J.F.R. Archilla ${ }^{a}$ \\ a Departamento de Física Aplicada, Universidad de Sevilla, P.O. Box 1065, Seville, Spain \\ b Departamento de FAMN, Universidad de Sevilla, P.O. Box 1065, Seville, Spain \\ c Departamento de Matemáticas, Universidad de Cádiz, P.O. Box 40, Puerto Real (Cádiz), Spain
}

Received 16 November 1994; revised manuscript received 24 March 1995; accepted for publication 28 March 1995

Communicated by A.P. Fordy

\begin{abstract}
We report on a phenomenon observed in a driven nonlinearly damped oscillator when two control parameters, the frequency of the external excitation and the nonlinear damping coefficient, are varied simultaneously. An interior crisis locus and a boundary crisis locus, corresponding to two different chaotic attractors, intersect in a point of the parameter space. There exists an interchange in the type of crisis that each attractor suffers after crossing the intersection point.
\end{abstract}

\section{Introduction}

In the theory of nonlinear dissipative systems studies are usually made by changing only one single control parameter. The majority of the physical systems involves two or more of these parameters and a great variety of phenomena may appear when more than one of them is varied simultanously.

One of the most interesting aspects which should be considered in the study of nonlinear dissipative systems involving two varying control parameters is the crisis locus, i.e., the locus of those parameters where boundary crises or interior crises occur [1]. A boundary crisis appears when a chaotic attractor collides with an unstable orbit which lies on the boundary of its basin of attraction and then disappears. An interior crisis appears when a chaotic attractor collides with an unstable orbit, which is in the interior of its basin, and suffers a sudden change in size. To determine a crisis locus one has to vary two parameters simultaneously, one is needed to assure the existence of the crisis and the other one is used to follow the evolution of the crisis in the parameter space.

Among the nonlinear systems that have been the object of more extensive studies are nonlinear driven oscillators [2-4], exhibiting a great richness of phenomena, crises among them [5,6]. A model system in which these last phenomena appear is that corresponding to the description of current-fed Josephson junctions taking into account interference effects, or its mechanical analogous, a driven nonlinearly damped pendulum $[7,8]$.

This model has four control parameters, one of them is related to the nonlinear damping and another refers to the frequency of the external excitation. The purpose of this paper is to report on some phenomena occurring when these two parameters are varied simultaneously. An interior crisis locus and a boundary crisis locus corresponding to two different attractors appear, they intersect in a point of the parameter space where two crises occur simultaneously. There is an interchange of the type of crisis that each attractor suffers after crossing this point. 


\section{Model equations}

The nonlinear evolution equations that are the object of our study read

$\frac{\mathrm{d} X_{1}}{\mathrm{~d} t}=X_{2}$

$\frac{\mathrm{d} X_{2}}{\mathrm{~d} t}=-\alpha\left(1+\gamma \cos X_{1}\right) X_{2}-\sin X_{1}+I \sin \omega t$,

where $\alpha, \gamma, I, \omega$ are control parameters.

These equations describe the dynamics of a driven pendulum with nonlinear damping effects. They are adequate to model the evolution of a Josephson junction under the excitation of a radio frequency current, and in this case the variable $X_{2}$ rcpresents the difference of the potential between the two superconductors of the junction. The term $\gamma \cos X_{1}$ represents the interference effects between the quasiparticle-pair and quasiparticle currents, which is usually called " $\cos \phi$ " term. Most work on driven Josephson tunneling junctions has neglected this interference term and some problems persist in relation to it, for example, the existing theories predict relative signs of the quasiparticle and quasiparticle-pair currents which are in contradiction with some experimental results [9].

In previous works $[7,8]$ we considered the interference term with a value for $\gamma$ in the range supported by the experimental results. Our results showed that its inclusion gives rise to a substantial modification of the dynamics as, for example, the appearance of a boundary crisis and of interior crises involving brokensymmetric chaotic attractors, etc.

In our study we have chosen $\alpha$ and $I$ as fixed parameters, with the values $\alpha=0.4, l=0.8$, and as varying parameters we have chosen $\gamma$ in the range $-1<\gamma<$ 1 , and $\omega$ in the range $0.1<\omega<1$. Within these ranges we can find possible sets of representative values for a Josephson junction [10]. Eqs. (1) and (2) have been resolved in double precision arithmetic using a fourth-order variable-step-size Runge-Kutta method. Poincare sections have been obtained by sampling the solutions once every period of the forcing term.

\section{Crisis loci}

In a previous paper [8] we showed the existence of a boundary crisis for $\omega=\omega_{c} \simeq 0.665, \gamma=-0.8$,

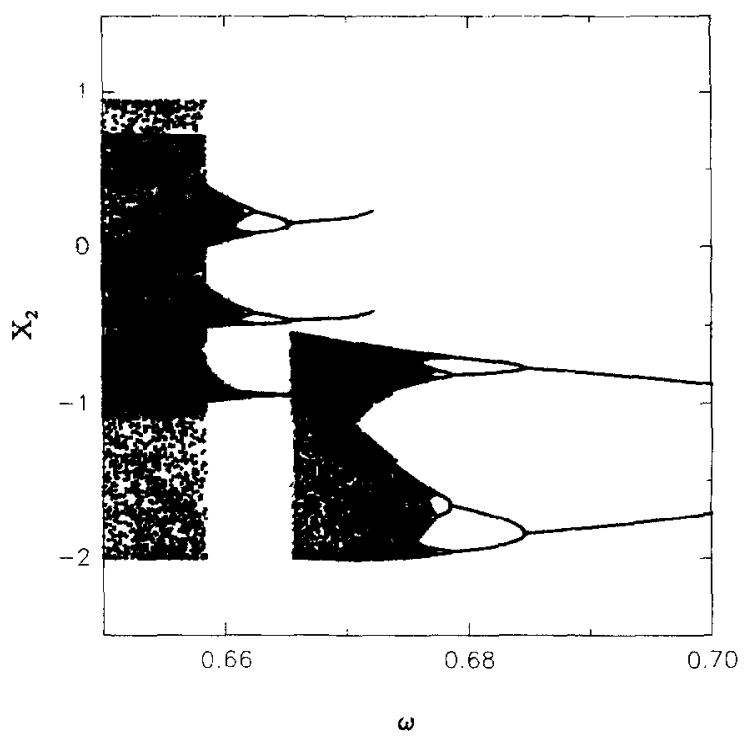

Fig. 1. Global bifurcation diagram for the variable $X_{2}$ with $\gamma=-0.8$.

$\alpha=0.4$ and $I=0.8$. This phenomenon consists in the collision of a chaotic attractor with an unstable periodthree orbit located on the basin boundary, originating a discontinuous disappearance of this chaotic attractor. Fig. 1 shows the bifurcation diagram corresponding to the variable $X_{2}$ for $\gamma=-0.8$. Previous to this crisis we can appreciate the coexistence of two attractors, a chaotic attractor and a stable period-three orbit.

Two accessible period-three saddle orbits [11] exist, one of them is accessible from the basin of attraction of the chaotic attractor and the other one is accessible from the basin of attraction of the period-three orbit. In Fig. 2 we show two-dimensional Poincaré sections of all the attractors which exist for $\omega=0.666$, the largest full circles represent an unstable periodthree orbit that was created for larger values of $\omega$, the smaller full circles and the open circles represent, respectively, the stable and the unstable period-three orbit created after a saddle-node bifurcation. We have superimposed the basins of attraction of the coexistent attractors. We represent in blank the basin of the chaotic attractor and with black points the basin of attraction of the period-three orbit. We can see that the chaotic attractor is about to collide with an accessible saddle orbit, announcing a boundary crisis. After the collision, points of the chaotic attractor will eventually come close to the saddle point, passing it along the 


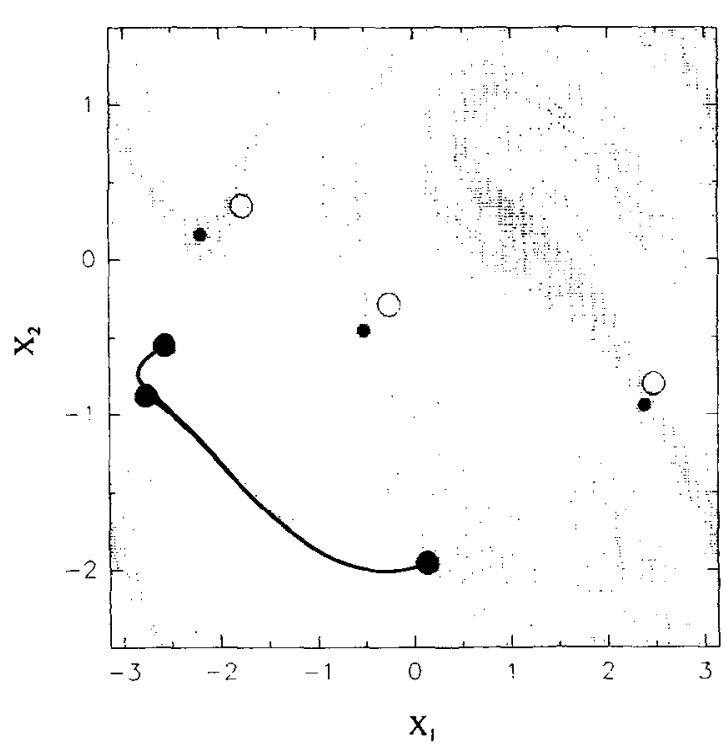

Fig. 2. Basins of attraction in the plane $\left(X_{1}, X_{2}\right)$ for the chaotic solution (blank) and the coexistent period-three solution (shaded), for $\omega=0.666$ and $\gamma=-0.8$. Also shown are the Poincaré sections of the chaotic attractor, its accessible saddle orbit (centers of the largest full circles), the period-three orbit (centers of the smallest full circles) and its associated accessible saddle orbit (centers of the open circles).

unstable orbit which lies in the basin of attraction of the stable period-three attractor, and then the chaotic attractor is destroyed.

By decreasing $\omega$ below $\omega_{\mathfrak{c}}$, the remaining attractor suffers a sequence of bifurcations creating a threepiece chaotic attractor. This attractor ultimately suffers an interior crisis when it collides with the unstable orbit created at the saddle-node bifurcation. Fig. 3 shows two-dimensional Poincaré sections prior to this interior crisis.

A study similar to the previous one may be done for differents values of the parameter $\gamma$. In this way it is possible to construct the crisis loci corresponding to the two types of crises just mentioned. We have considered the interval of $\gamma$ values $-1<\gamma \leqslant-0.8$, which is in the range supported by experimental results in Josephson junctions [12]. Exploration with values of $\gamma$ larger than -0.8 is not necessary, since the boundary crisis soon disapear and there is no crisis locus. Fig. 4 shows two different crisis loci corresponding to the two coexistent chaotic attractors. The two curves intersect in a point located roughly at $(\gamma, \omega) \simeq$ $(-0.88,0.669)$. In Fig. 5 we show the phenomenon

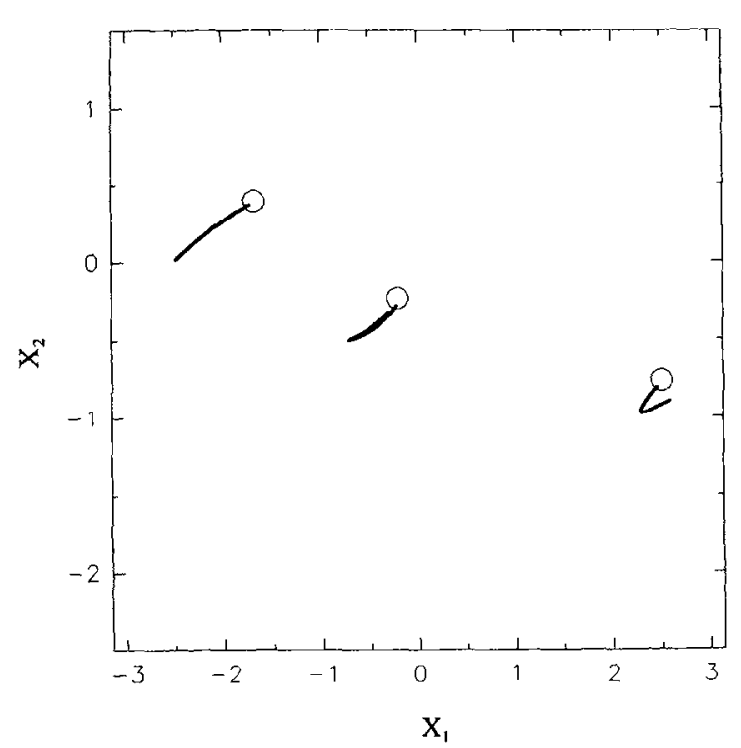

Fig. 3. Two-dimensional Poincaré sections for $\omega=0.659$ and $\gamma=-0.8$. The centers of the open circles represent the unstable orbit which is close to colliding with the three-piece chaotic attractor.

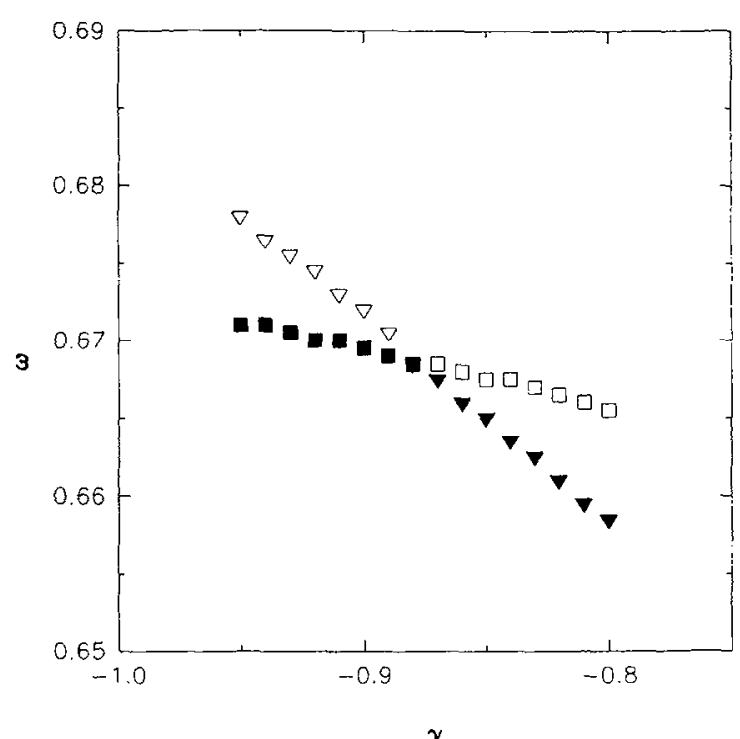

Fig. 4. Crisis loci in the plane $(\gamma, \omega)$. The centers of the squares represent the crisis locus of the three-piece chaotic attractor, and the centers of the triangles the crisis locus of the other chaotic attractor. Open symbols indicate interior crises and full symbols boundary crises. 


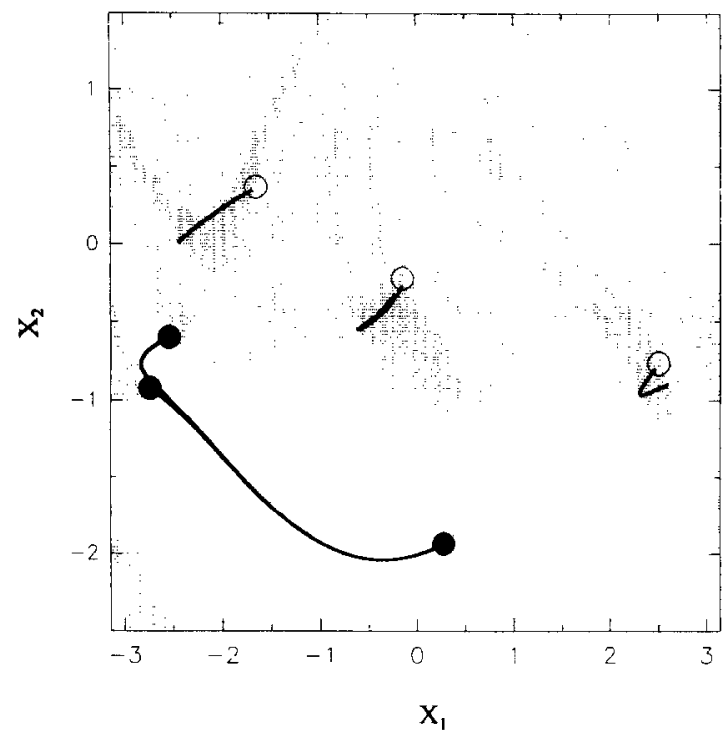

Fig. 5. Basins of attraction in the plane $\left(X_{1}, X_{2}\right)$ for the one-piece chaotic attractor (blank) and the coexistent three-piece chaotic attractor (shaded), for $\omega=0.6695$ and $\gamma=-0.88$. Also shown are the Poincare sections of these two chaotic attractors and their accessible saddle orbits (centers of the full circles and centcrs of the open circles, respectively).

of simultaneous coincidence of crises. We have represented the basins of attraction of the two coexistent chaotic attractors, their Poincaré sections and their accessible saddle orbits. The stable manifolds of each accessible saddle orbit are situated along the borderline of the basin boundary, and the two chaotic attractors lic on the closurc of their respective unstable manifolds. As crises are approached both attractors collide with their respective accessible orbit, their stable and unstable manifolds become tangent and cross after collision. Then, points of each attractor pass along the boundaries appearing only one large chaotic attractor. This jump in size can be appreciated in the global bifurcation diagram represented in Fig. 6.

As $\gamma$ decreases below this critical value, there exists an interchange in the type of crisis that each attractor suffers, that is, the three-piece attractor undergoes boundary crises, and the one-piece attractor interior crises. This new behavior of the system can be visualized in the global bifurcation diagram of Fig. 7.

By considering the crisis locus of each attractor separately, the intersection point delimits two branchs, one corresponds to interior crises and the other one to

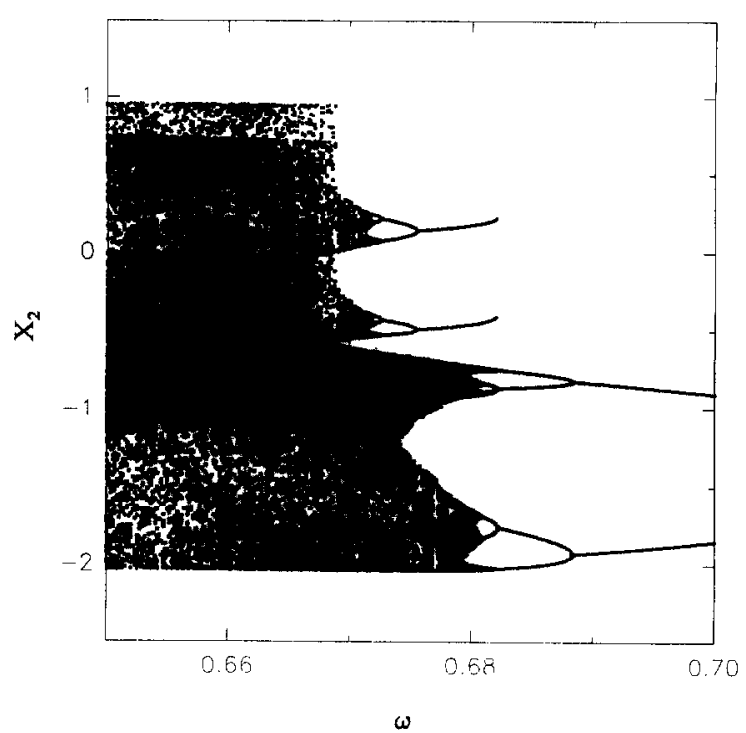

Fig. 6. Global bifurcation diagram for the variable $X_{2}$ with $\gamma=-0.88$. For this value of $\gamma$ two crises occur simultaneously.

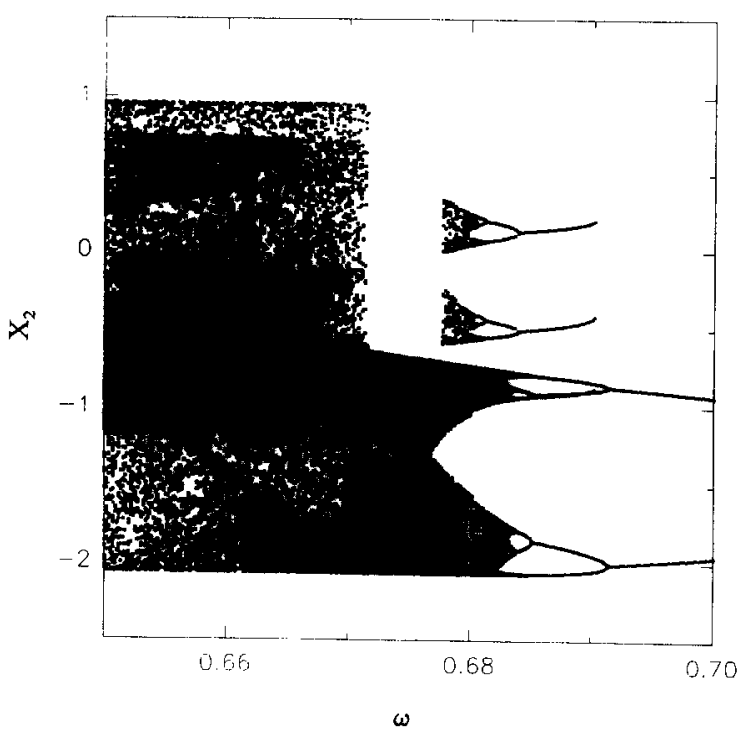

Fig. 7. Global bifurcation diagram for the variable $X_{2}$ with $\gamma=-0.95$

boundary crises. This point is not a vertex in the sense of Gallas, Grebogi and Yorke [13], that is, a point of a boundary crisis locus where a chaotic attractor undergoes an interior crisis and simultaneously its basin suffers a metamorphosis [11]. Similar phenomena, involving changes in accessible boundary orbits, have 
been found in a twin-well Duffing oscillator [14].

\section{Conclusions}

In this paper we have described, by analyzing the parameter space of a driven nonlinearly damped oscillator, the dynamics along crisis loci. We have found that two crises loci, corresponding to two differents chaotic attractors, intersect in a point of the parameter space. At this critical point both attractors interchange the type of crisis they suffer.

Our study has been restricted to situations involving just two parameters. It is expected that for more than two parameters these types of phenomena will be more complex.

\section{References}

[1] C. Grebogi, E. Ott and J. A. Yorke, Physica D 7 (1983) 181.

[2] S. Sato, M. Sano and Y. Sawada, Phys. Rev. A 28 (1983) 1654.
[3] V. Parlitz and W. Lauterborn, Phys. Rev. A 36 (1987) 1428.

[4] D. D'Humieres, M.R. Beasley, B.A. Huberman and A. Libchaber, Phys. Rev. A 26 (1982) 3483.

[5] B. Wu and J.A. Blackbum, Phys. Rev. A 45 (1992) 7030.

[6] W.L. Ditto, S. Rauseo, R. Cawley, C.Grebogi, G.-H. Hsu, E. Kosterlich, E. Ott, H.T. Savage, R. Segnan, M.L. Spano and J.A. Yorke, Phys. Rev. Lett. 63 (1989) 923.

[7] F. Palmero and F. Romero Romero, Phys. Lett. A 160 (1991) 553.

[8] F. Palmero and F. Romero Romero, Phys. Lett. A 179 (1993) 337.

[9] A. Barone and G. Paterno, Physics and applications of the Josephson effect (Wiley, New York, 1982).

[10] N.F. Pedersen, J. Appl. Phys. 44 (1973) 5120.

[11] C. Grebogi, E. Ott and J.A. Yorke, Physica D 24 (1987) 243.

[12] N.F. Pedersen, T.F. Finnegan and D.N. Langenberg, Phys. Rev. B 6 (1972) 4151.

[13] J.A.C. Gallas, C. Grebogi and J.A. Yorke, Phys. Rev. Lett. 71 (1993) 1359.

[14] Y. Ueda, S. Yoshida, H.B. Stewart and J.M.T. Thompson, Philos. Trans. R. Soc. A 332 (1990) 169. 